\title{
The Sunyaev-Zeldovich effect from clusters of galaxies
}

\author{
Etienne Pointecouteau ${ }^{1, *}$ \\ ${ }^{1}$ IRAP, Université de Toulouse, CNRS, CNES, UPS, (Toulouse), France
}

\begin{abstract}
In this paper, we recall the basics of the the Sunyaev-Zeldovich effect from groups and clusters of galaxies. We review the transformational results from SZ surveys in the past decade, that have led to the detection of new clusters of galaxies from the local to the very distant Universe. The SZ effect has become a very efficient way to investigate the astrophysics of the hot intra-cluster gas, very competitive and complementary to X-ray observations. It renewed the use of massive halos as a cosmological probe or to study the physics of structure formation and evolution. We discuss the present strong synergies between the SZ and X-ray observations.
\end{abstract}

\section{Introduction}

The inverse Compton (IC) interaction of the hot gas in massive halos, i.e., groups and clusters of galaxies, produced a secondary anisotropie in the cosmic microwave spectrum. This effect was first theorised by Sunyaev and Zeldovich [1], hence its eponym name. Its first detection [2] happened soon after the first detection of the X-ray emission from the hot galaxy cluster atmosphere [3]. X-ray observation of massive halos met its golden age with space missions such as Ginga, ROSAT or ASCA, followed by the highly productive and still operating satellites XMM-Newton and Chandra. Meanwhile, SZ observations have lagged behind due mainly to technological limitations, and SZ measurements have been relatively sparse. The last two decades have seen the drastic advent of CMB observations, hence this of galaxy clusters through the SZ effect. Surveys like ACT [4], SPT [5] and the Planck [6] space mission, initially mainly designed to better understand the cosmology of our Universe through the CMB fluctuations in intensity and polarisation as main probe [e.g., 7], have revolutionised our vision of the population of massive halos, producing catalogues of new discovered clusters (see next section). Beyond a few pioneering observations with high spatial resolution [e.g., 8, 9], a new generation of ground-based instruments such as IRAM-30m/NIKA-2, GBT/MUSTANG-2 or ALMA now allow the detailed mapping of the SZ effect.

The aforementioned SZ effect, also called the thermal SZ effect (tSZ) arises from the ICM of groups and clusters of galaxies, but also from all structures embedding heated optically thin plasmas. The integrated effect produced by large scale structures is commonly referred as the $y$ spectral distorsion of the CMB spectrum [10]. The differential spectrum with respect to the $\mathrm{CMB}$ reference spectrum has a spectral characteristic shape, depleting through IC scattering the low frequency part of the CMB spectrum to populate the higher frequency end. This characteristic spectral has a dependence to the gas temperature at a second order level. This is known as the relativistic SZ effect due to the weakly relativistic tail of the electron

\footnotetext{
*e-mail: etienne.pointecouteau@irap.omp.eu
} 
Maxwellian velocity distribution [e.g., 11, 12]. Another effect is due to the peculiar global movement of the cluster halo in a referential comoving with the expansion of the Universe. This kinetic SZ (kSZ) effect has a Doppler signature as the CMB and is about an order of magnitude lower than the tSZ in amplitude [13]. Other subdominant SZ signals arise from polarisation [e.g., 13], non-thermal populations of electron in the ICM [e.g., 14] or a low probability for multiple IC scattering [13]. These latest processes are not considered in the following. We refer the reader to the recent review by [15] for extensive discussions on these subjects. The SZ brightness is not prone to the usual redshift deeming, although the SZ flux is through its dependency with the solid angle over which it is measured (i.e., the dilution by the instrumental beam).

The relative monochromatic surface brightness of the SZ effect with respect of these of the $\mathrm{CMB}$ can then be formalised as follow:

$$
\frac{\Delta I_{v}}{I_{v}}=y_{t} f\left(v, T_{e}\right)+y_{k} f\left(v, T_{e}, v_{p}\right)
$$

with

$$
y_{t}=\frac{\sigma_{T}}{m_{e} c^{2}} \int_{l o s} P_{e} d l
$$

and

$$
y_{k}=-\frac{\sigma_{T}}{c} \int_{l o s} v_{p} n_{e} d l
$$

where $\sigma_{T}, m_{e}$ and $c$ are the Thomson cross-section, mass of the electron and speed of light, respectively. $T_{e}, P_{e}$ and $n_{e}$ are the temperature, thermal pressure and density of the ICM, respectively. $v_{p}$ is the peculiar velocity of the comoving gas.

The spectral shape and dependencies in gas temperature and velocity are illustrated on Fig 1.

\section{SZ surveys}

Three survey instruments have been efficiently operated in the past two decades at millimetre wavelengths: the Atacama Comology Telescope, the South Pole Telescope and the Planck satellite. The three were designed to primarily study the anisotropies of the CMB, however they were also optimise to detect the SZ effect from clusters of galaxies. As a consequence they produced the first catalogues of galaxy clusters since RASS. With their spatial resolution of 1.5, 1.6 and 4.5 to 10 arcmin FWHM (at the wavelengths relevant for the detection of the SZ effect), they produced catalogues with 91, 747 and 1963 entries including newly detected SZ clusters, respectively [16-20].

With its all-sky coverage the Planck survey has sampled the Universe's whole volume, although with moderate spatial resolution, allowing to detect all the most massive clusters (i.e., $M_{500}>5 \times 10^{14} \mathrm{M}_{\odot}$ ) in the local Universe and out to intermediate redshifts, including very extended nearby clusters such as Virgo [21] or Coma [22]. The ACT and SPT surveys nicely complemented this approach thanks to their higher spatial resolution allowing a deeper investigation of the cluster population towards higher redshifts and lower masses [i.e., $z>1$ and $M_{500}>2-3 \times 10^{14} \mathrm{M}_{\odot}$. See e.g., 23].

The three experiments have delivered has expected a wealth of results on the population of clusters, used as complementary probe to the CMB for cosmological studies [e.g., 2427] and to better understand the physics of the intra-cluster medium in massive halos [e.g., 28-30]. 


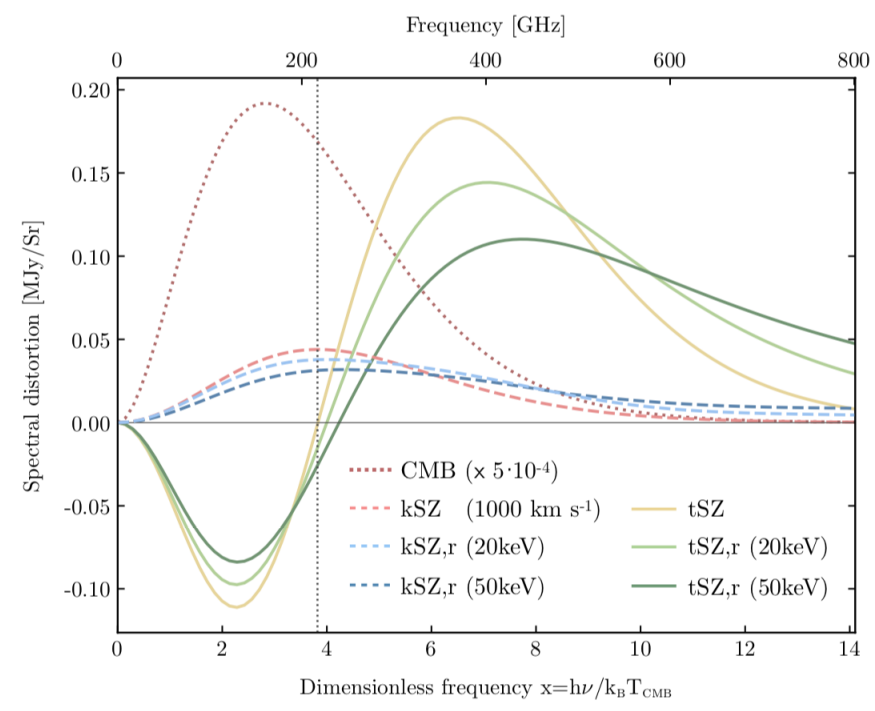

Figure 1. Figure reproduced from Fig 2 in [15], quoting the afferent caption: "Thermal (solid) and kinematic (dashed) SZ spectra, including relativistic corrections for various temperatures. We assumed an optical depth $\tau_{e}=10^{-2}$ and an overall Compton parameter $y=10^{-4}$. The dotted, dark red curve illustrates the shape of the unscattered CMB spectrum, which for comparison was scaled by a factor of $5 \times 10^{-4} . "$

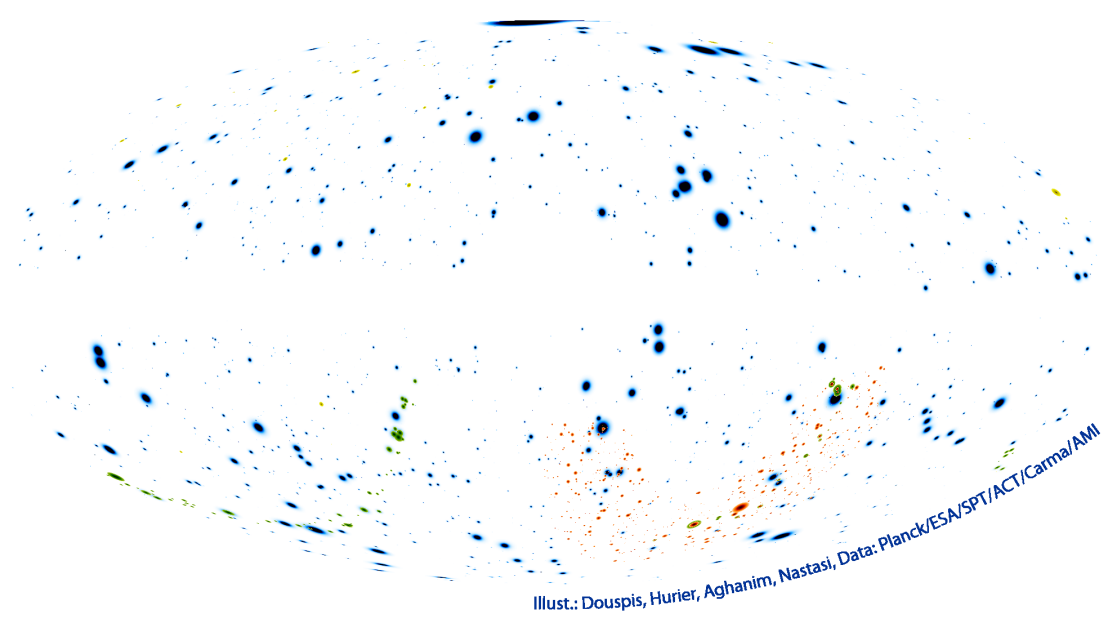

Figure 2. Distribution over the sky in galactic gnomonic projection of the ACT(green), SPT (red), Planck (blue) clusters . Credits: Doupis et al., The SZ Cluster Database project, http://szcluster-db.ias.upsud.fr/

\section{Cluster physics from the SZ and X-ray synergy}

Synergies with other observables at other wavelengths have been key to the aforementioned results. X-rays likely come first among those. The SZ effect and the X-ray emis- 
sion from groups and clusters trace the same physical component, the hot gas. The thermal SZ effect is directly proportional to the integrated thermal pressure along the line of sight. The X-ray brightness is a function of both gas temperature and density, i.e., $I_{X} \propto \int n_{e}^{2} \times \sqrt{k T} \times \exp (-1 / k T)$, and its dependance in temperature is rather mild in the low energy band (i.e., $E \lesssim 2 \mathrm{keV}$ ) at least for clusters with ICM temperature above $2-3 \mathrm{keV}$. The two observables are therefore extremely complementary. They provide a very consistent view of the gas content of groups and clusters as illustrated by several results [e.g., 31, 32], making their combination even more powerful.

Assuming a given cosmology, the SZ and X-ray signals provide the thermal pressure and density of the gas, respectively. All other thermodynamical properties (e.g., temperature, entropy, hydrostatic mass) can be derived from their combination. This method has been initially applied to determine the global ICM temperature of RX J2228+2037 from Nobeyama$45 \mathrm{~m} 22 \mathrm{GHz}$ and ROSAT-HRI observations [33]. Kitayama et al. [34] first extended the method to structural properties, deriving the temperature profile of of RX J1347-1145 from $N o B a$ and Chandra data. The study of X-ray and SZ scaling relations and structural properties have extensively taken advantage of this technique, allowing to firmly establish the self-similar distribution of the hot ICM gas in pressure, entropy, mass, gas fraction in nearby clusters [e.g., 28, 35-39]. This methodology is now being extended to higher redshifts in order to assess the impact of baryons physics on the evolution of cluster properties across cosmic times. A prerequisite to maximise the efficiency of such investigation is for the spatial resolution in the X-ray and SZ to be equivalent. Although this was not the case for SZ surveys, the new generation of high resolution SZ instruments offer spatial resolution going from 18" [NIKA-2, e.g., 40], 9" [Mustang-2, e.g., 41] down to 5" [ALMA, e.g., 42]. The association of these data with X-ray observations has demonstrated the applicability of the aforementioned method to high redshift systems [43-45].

Beyond the fiducial radius $R_{500}{ }^{1}$, the X-ray emission drops drastically and bring the addition of the SZ information to its full strength. The characterisation of the thermal pressure distribution from the cluster centre to the outskirts has been tackled through joint $\times \mathrm{MM}$ Newton and Planck analysis out to radii of $\sim 1.5-2 R_{500}$ for individual clusters and of $\sim 3 R_{500}$ from statistical analysis $[28,38]$. These regions are the locus of gas accretion from the larger cosmic web environment and of the gas virialisation [see 46, for a review]. Concomitant radial coverage from X-ray and SZ measurements allows to look into the induced complex gas dynamics, which involves thermal and non-thermal virialisation, or generation of turbulence and large scale bulk motions [47-49].

Figure 3 illustrates the above, and presents the reconstruction of the temperature profiles from the combination of XMM-Newton and NIKA-2 data combination [44] and the multiwavelength analysis of MACS J0717.5+3745 including the first mapping of the kinetic SZ effect from the NIKA-2 measurements.

The dynamics of the intra-cluster gas can also be looked at through joint X-ray and SZ analysis. The combination of XMM-Newton and Planck emission have led to the characterisation of shocks in the Coma cluster through the detection of edges consistently seen at both wavelengths [22]. One is also seen in radio. This coincidence allows to study re-acceleration processes at play in the cluster outskirts, through constraints on the magnetic field strength or/and on turbulence/shock waves. Such features can be investigated in high redshift clusters with high resolution SZ and X-ray observations, as achieved in MACS J0744.9+3927 [ $z \sim 0.7,51]$ or the El Gordo cluster $[z \sim 0.9,52]$. Similarly, following the method first developed by Churazov et al. [53], Khathi \& Gaspari [54] have investigated the statistics of

\footnotetext{
${ }^{1}$ Radius encompassing a density contrast of 500 with respect to the critical density of the Universe at the cluster's redshift.
} 

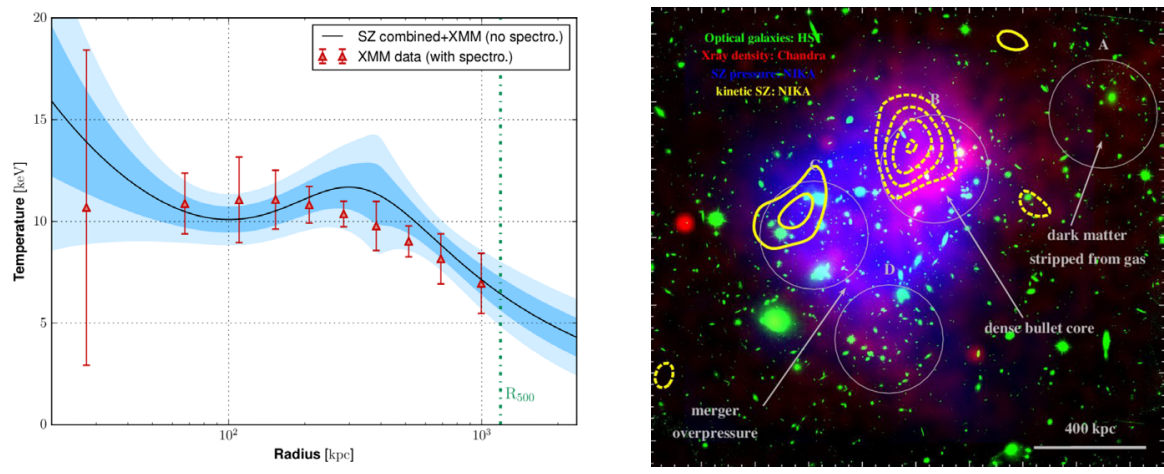

Figure 3. (left) Deprojected radial temperature profiles of PSZ2 G144.83+25.11( $z=0.58)$ reconstructed from the combination of the gas pressure profile derived from NIKA-2 data and the gas density profile obtained from the XMM-Newton observation. The red points trace the profile obtained from the X-ray spectroscopy. The dark and light blue regions show $68 \%$ abs $95 \%$ error envelops. The best fit is showns by a black solid line [reproduced from Fig 11 in 50]. (right) Multi-wavelength image of MACS J0717.5+3745 showing the galaxy distribution (green, Hubble Space Telescope), the electron gas density (red, Chandra X-ray data), the pressure (blue, NIKA) and the kSZ signal (yellow contours, NIKA, figure below). The gray circles indicate the main sub-clusters [see 45 , for details].

surface brightness fluctuations as a probe of the underlying bulk motion/turbulent velocity field in order to constraint the pressure fluctuations at larger (injection) scales and thus assess the dynamic state of the Coma ICM (i.e., in adiabatic mode). The combination of the X-ray and SZ images offers also the possibility to perform 2D studies and push further the investigation of the internal dynamics and structure of clusters. For instance, the massive triple merger cluster, MACS J0717.5+3745 at $z=0.55$, has first been looked at with Mustang, Bolocam and Chandra data in order to probe the motions of its various components [55]. More recently, Adam et al. [45, 56] have reconstructed the first map for this cluster of the kinetic SZ effect and of the gas temperature, underlying the thermodynamics of the ICM and of its substructures from the combination of the NIKA SZ map and the XMM-Newton temperature map. At the very center of clusters, the physics of the AGN feedback can also be probed from the SZ effect in conjunction with X-ray data [57].

\section{Perspectives}

The future of SZ surveys lies on the ground with ACT-Pol [58] and SPT-3G [59]. They will produce large catalogues sampling deeper in redshift and lower in mass the population of groups and clusters of galaxies (e.g., SPT-3G should allow to go down to $\sim 10^{14} \mathrm{M}_{\odot}$ and out to $z=1.5$ ). The synergy with the upcoming eROSITA X-ray survey is obvious and shall lead to a better statistical understanding of the population of massive halos. We need to fully understand how matter assembles within structure across cosmic time, what is the impact of baryons in the formation and the evolution of massive halos, and how their physics link at various scales, i.e., from the wider ICM to galaxies and their hosted super massive black holes [see e.g., 60]. As aforementioned, the SZ measurements can play a major role here on the observational side, through its powerful synergy with X-rays and especially towards the high redshift Universe, in order to unveil whether the baryonic and dark matter component actually follow the evolution expected from the hierarchical model of structure formation. The spatial resolution demonstrated by NIKA-2, Mustang- 2 and ALMA are now comparable to these of 
Chandra and XMM-Newton in X-rays. They have redeemed the potential of pointed SZ observations with single dish and interferometer telescopes, and open thereby a new bright era for SZ cluster observations.

We redirect the reader to the recent review on the SZ effect by Mroczkowski et al. [15] for further comprehensive reading.

I am grateful to the organisers of the conference "mm Universe @ NIKA2: Observing the millimeter Universe with the NIKA2 camera" held in Grenoble, France, on $3^{\text {rd }}-7^{\text {th }}$ of June 2019 for their invitation to present this paper.

\section{References}

[1] R.A. Sunyaev, Y.B. Zeldovich, Comments on Astrophysics and Space Physics 4, 173 (1972)

[2] Y. Pariysky, Sov. Astron. Lett.-JETP 4, 730 (1973)

[3] H. Gursky et al., ApJ (Lett.)167, L81 (1971)

[4] D.S. Swetz et al., ApJ Suppl.194, 41 (2011), 1007.0290

[5] J.E. Carlstrom et al., P. A. S. P.123, 568 (2011), 0907.4445

[6] Planck Collaboration, $A \mathcal{E} A 536$, A1 (2011), 1101.2022

[7] Planck Collaboration, AEFA596, A108 (2016), 1605.03507

[8] E. Pointecouteau, et al., ApJ552, 42 (2001)

[9] E. Komatsu et al., PASJ53, 57 (2001), astro-ph/0006293

[10] J. Chluba, R.A. Sunyaev, MNRAS419, 1294 (2012), 1109.6552

[11] S.Y. Sazonov, R.A. Sunyaev, ApJ508, 1 (1998)

[12] E. Pointecouteau, M. Giard, D. Barret, $A \mathcal{E} A 336,44$ (1998), astro-ph/9712271

[13] R.A. Sunyaev, I.B. Zeldovich, MNRAS190, 413 (1980)

[14] T.A. Enßlin, C.R. Kaiser, AEA A360, 417 (2000), astro-ph/0001429

[15] T. Mroczkowski et al., Space Sci. Rev.215, 17 (2019), 1811.02310

[16] M. Hasselfield et al., JCAP7, 008 (2013), 1301.0816

[17] L.E. Bleem et al., ApJ Suppl.216, 27 (2015), 1409.0850

[18] Planck Collaboration 2011, AEFA536, A8 (2011), 1101.2024

[19] Planck Collaboration, $A \mathcal{F} A 571$, A29 (2014), 1303 . 5089

[20] Planck Collaboration, AEA A594, A27 (2016), 1502.01598

[21] Planck Collaboration, $A \mathcal{E} A$ 596, A101 (2016), 1511.05156

[22] Planck Collaboration, $A \mathcal{E} A 554$, A140 (2013), 1208. 3611

[23] M.B. Bayliss et al., ApJ794, 12 (2014), 1307.2903

[24] J.L. Sievers et al., JCAP10, 060 (2013), 1301.0824

[25] T. de Haan et al., AEFA594, A24 (2016)

[26] Planck Collaboration, $A \mathcal{E} A 594$, A24 (2016), 1502.01597

[27] Planck Collaboration, $A \mathcal{F} A 571$, A20 (2014), 1303.5080

[28] Planck Collaboration, $A \mathcal{E} A 5550$, A131 (2013), 1207.4061

[29] F. Menanteau et al., ApJ765, 67 (2013), 1210.4048

[30] M. McDonald et al., ApJ794, 67 (2014), 1404.6250

[31] Planck Collaboration, $A \mathcal{E} A 536$, A10 (2011), 1101.2043

[32] Planck Collaboration, $A \mathcal{E} A 536$, A11 (2011), 1101.2026

[33] E. Pointecouteau et al., $A \mathcal{E} A 387,56$ (2002), astro-ph/0203268

[34] T. Kitayama et al., PASJ56, 17 (2004), astro-ph/0311574

[35] M. Nord et al., AEA506, 623 (2009), 0902.2131 
[36] K. Basu et al., AEA519, A29 (2010), 0911.3905

[37] T. Plagge et al., ApJ716, 1118 (2010), 0911.2444

[38] J. Sayers et al., ApJ832, 26 (2016), 1605.03541

[39] D. Eckert et al., AEGA551, A22 (2013), 1301.0617

[40] R. Adam et al., AEA A569, A66 (2014), 1310.6237

[41] P.M. Korngut et al., ApJ734, 10 (2011), 1010.5494

[42] T. Kitayama et al., PASJ68, 88 (2016), 1607.08833

[43] R. Adam et al., AEA A576, A12 (2015), 1410.2808

[44] F. Ruppin et al., AEA597, A110 (2017), 1607.07679

[45] R. Adam et al., AEA A598, A115 (2017), 1606.07721

[46] S. Walker et al., Space Sci. Rev.215, 7 (2019), 1810.00890

[47] V. Ghirardini et al., AEA621, A41 (2019), 1805.00042

[48] D. Eckert et al., AEA621, A40 (2019), 1805.00034

[49] S. Ettori et al., AEA621, A39 (2019), 1805.00035

[50] F. Ruppin et al., AEAA615, A112 (2018), 1712.09587

[51] A. Wilber et al., MNRAS476, 3415 (2018), 1802.06791

[52] K. Basu et al., ApJ (Lett.)829, L23 (2016), 1608.05413

[53] E. Churazov et al., MNRAS421, 1123 (2012), 1110.5875

[54] R. Khatri, M. Gaspari, MNRAS463, 655 (2016), 1604.03106

[55] T. Mroczkowski et al., ApJ761, 47 (2012), 1205.0052

[56] R. Adam et al., AEA606, A64 (2017), 1706.10230

[57] Z. Abdulla et al., ApJ871, 195 (2019), 1806.05050

[58] F. De Bernardis et al., in PROC SPIE. (2016), Vol. 9910, p. 991014, 1607.02120

[59] B.A. Benson et al., in PROC SPIE. (2014), Vol. 9153, p. 91531P, 1407.2973

[60] V. Springel et al., Nature435, 629 (2005), astro-ph/0504097 